\title{
Taxas de reganho ponderal em pacientes bariátricos acompanhados na Policlínica Piquet Carneiro
}

\author{
Weight regain in bariatric patients followed at \\ Policlínica Piquet Carneiro
}

\author{
Lutieska F. Garroni, Ana Carolina B. Bacellar, Diana Carla G. Lima, Juliana D’Augustin,
}

Vicente L. da Silva Júnior, Diogo G. Panazzolo, Bernard B. Barroso, Luiz Guilherme K. de Aguiar*

\begin{abstract}
Resumo
A obesidade é uma doença com alta prevalência em nosso meio e o tratamento cirúrgico pode promover redução da morbimortalidade cardiovascular. Apesar do maior conhecimento sobre as técnicas cirúrgicas empregadas e manejo clínico destes pacientes, ainda observam-se altas taxas de reganho ponderal. O objetivo deste estudo é avaliar a taxa de reganho (TxR) ponderal dos pacientes bariátricos atendidos em consulta de primeira vez na Policlínica Piquet Carneiro. Foram recrutados 35 pacientes (29 mulheres e 6 homens; 39,3 \pm 9,10 anos) submetidos à cirurgia bariátrica entre abril e setembro de 2013. O índice de massa corporal $\left(\mathrm{IMC}=\right.$ peso/altura $\left.{ }^{2}\right)$ foi calculado e, com base no relato do peso pré-cirúrgico e do peso mínimo alcançado após a cirurgia, foram calculados respectivamente, o IMC pré-cirúrgico e o IMC mínimo. A TxR foi calculada [(peso atual - peso mínimo relatado) x 100/(peso pré-operatório relatado - peso mínimo relatado)]. Os pacientes foram divididos conforme o tempo decorrido da cirurgia, primeiramente em dois grupos: $\leq 1$ ano $(G \leq 1)$ e $>1$ ano $(G>1)$. Posteriormente, estes foram reagrupados em outros dois novos grupos: $<2$ anos $(G<2)$ e $\geq 2$ anos $(G \geq 2)$ e ainda em $<5$ anos $(G<5)$ e $\geq 5$ anos $(G \geq 5)$. Dos 35 pacientes recrutados, 94,3\% $(n=33)$ relataram a realização de gastroplastia redutora com reconstrução em $Y$ de Roux. O IMC pré-cirúrgico relatado era de $50,5 \pm 8,92 \mathrm{~kg} / \mathrm{m}^{2}$. Em relação ao tempo decorrido da cirurgia, observamos que $22,8 \%(n=8)$ da amostra havia realizado a cirurgia em até um ano, $40 \%(n=14)$ em menos de dois anos e 74,3\% $(n=26)$ em menos de cinco anos. O IMC mínimo alcançado foi de $30,7 \pm 6,6 \mathrm{~kg} / \mathrm{m}^{2}$. O percentual de pacientes que apresentavam reganho ponderal foi de $71,4 \%(\mathrm{n}=25)$ e o

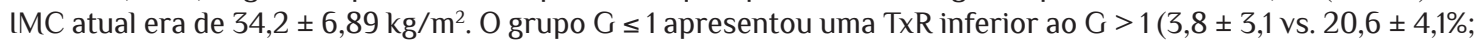
$p<0,05)$. Tal fato se acentua após o segundo ano da cirurgia ( $2,6 \pm 1,8$ vs. $26,2 \pm 4,6 \%$; $><0,001)$; tornando-se ainda mais relevante após cinco anos da cirurgia (10,1 3,0 vs. 35,9 $\pm 7,2 \% ; p<0,001)$. Conclui-se que o reganho ponderal já esteve presente no primeiro ano do pós-operatório, entretanto torna-se mais relevante em função do tempo decorrido da cirurgia.
\end{abstract}

Descritores: Obesidade; Cirurgia bariátrica; Ganho de peso; Prognóstico.

\begin{abstract}
Obesity is a disease with high prevalence in our country and surgical treatment may reduce cardiovascular morbimortality. Despite increased knowledge about surgical techniques and clinical management of these patients, we still observe high rates of weight regain. The aim of this study is to evaluate the rate of weight regain (TxR) of bariatric patients seen in a medical set for the first time at the Policlínica Piquet Carneiro for outpatients care. We recruited between April and September, 2013, 35 patients (29 females and 6 males; $39.3 \pm 9.10$ years) who have undergone bariatric surgery. The body mass index (BMI - weight in $\mathrm{kg} / \mathrm{height}^{2}$ ) was calculated and, based on the reported pre-surgical weight and minimum weight achieved after surgery, we calculated respectively the preoperative BMI and minimum BMI. The TxR was calculated [(current weight - minimum weight) $\times 100 /$ (weight preoperatively - minimum weight)]. The patients were divided according to the time from surgery, as follows: $\leq$ 1 year $(G \leq 1)$ and $>1$ year $(G>1)$. Afterwards, these patients were regrouped into two other new ones: $<2$ years $(G<2)$ and $\geq 2$ years $(G \geq 2)$ and further $<5$ years $(G<5)$ and $\geq 5$ years $(G \geq 5)$. Of the 35 patients enrolled, $94.3 \%(n=33)$ were submitted to Roux-en-Y gastroplasty. The preoperative BMI was reported to be $50.5 \pm 8.92$
\end{abstract}

*Endereço para correspondência: Ambulatório de Obesidade. Policlínica Piquet Carneiro. UERJ. Rua Marechal Rondon, 381, doi:10.12957/rhupe.2014.9812 
$\mathrm{kg} / \mathrm{m}^{2}$. Regarding the time of surgery, we observed that $22.8 \%(\mathrm{n}=8)$ of the sample had performed their surgery within 1 year, $40 \%(n=14)$ in less than 2 years and $74.3 \%(n=26)$ within 5 years. The achieved minimum BMI was $30.7 \pm 6.6 \mathrm{~kg} / \mathrm{m}^{2} .71 .4 \%(n=25)$ of the patients have already referred weight regain at the first visit and current BMI was $34.2 \pm 6.89 \mathrm{~kg} / \mathrm{m}^{2}$. Group $\mathrm{G} \leq 1$ showed lower TxR than $\mathrm{G}>1$ (3.8 $\left.\pm 3.1 \mathrm{vs.} 20.6 \pm 4.1 \%, \mathrm{p}<0.05\right)$. This was highlighted after the second year of surgery ( $2.6 \pm 1.8$ vs. $26.2 \pm 4.6 \%, p<0.001)$, making it even more relevant after five years from surgery $(10.1 \pm 3.0$ vs. $35.9 \pm 7.2 \%, p<0.001)$. It is concluded that the weight regain was already present in the first year after surgery, but becomes more important due to the time from surgery.

Keywords: Obesity; Bariatric surgery; Weight gain; Prognosis.

\section{Introdução}

A obesidade é uma condição complexa, crônica e multifatorial, que apresenta, nos últimos anos, elevado crescimento em sua prevalência tanto em crianças e adolescentes quanto em adultos. Considerada atualmente uma pandemia, é um dos principais contribuintes para o aumento da prevalência de doenças crônicas não comunicáveis, como diabetes mellitus tipo 2 (DM2), hipertensão arterial sistêmica (HAS), dislipidemias e doenças cardiovasculares, gerando maior morbimortalidade e redução da qualidade de vida. ${ }^{1}$ Dados do Instituto Brasileiro de Geografia e Estatística (IBGE) de 2010 revelam excesso de peso em $48 \%$ de mulheres e em 50,1\% de homens adultos brasileiros. ${ }^{2}$

Uma redução de 5 a 10\% no peso, através de fármacos e/ou modificações de estilo de vida, está associada à melhora significativa de comorbidades cardiovasculares e metabólicas, com benefício principalmente para pacientes com obesidade graus I e II [índices de massa corporal (IMC) entre 30 e $39,9 \mathrm{~kg} / \mathrm{m}^{2}$ ]. Entretanto, em pacientes com obesidade grau III (IMC $\geq 40 \mathrm{~kg} / \mathrm{m}^{2}$ ), tais medidas, apesar de também promoverem benefício cardiometabólico, são em geral limitadas. Nestes casos, a cirurgia bariátrica pode trazer maior benefício, promovendo perda ponderal maior e sustentada, além de melhora ou resolução de complicações metabólicas e ainda redução da morbimortalidade cardiovascular associadas à obesidade. ${ }^{3}$ São candidatos ao tratamento cirúrgico aqueles pacientes com IMC grau III ou ainda aqueles com grau II quando associado à comorbidades, como apneia do sono, DM2, HAS, dislipidemia, dificuldades de locomoção, entre outras comorbidades de difícil manejo clínico. ${ }^{4}$

Apesar dos benefícios observados para redução do excesso de peso com a cirurgia bariátrica, o reganho ponderal é observado em subgrupos de pacientes, com percentual estimado de 10 a $20 \%$ do peso perdido durante o seguimento em longo prazo. Entretanto, há ainda limitações para predizer quais pacientes estão mais propensos a apresentar reganho, bem como qual a conduta nesses casos. Possíveis preditores do reganho incluem modificações comportamentais, desde transtornos compulsivos alimentares à redução de bem-estar. ${ }^{5}$ Há, portanto, necessidade de maior entendimento do processo de reganho de peso nos pós-operatórios para melhor direcionamento terapêutico.

O objetivo deste estudo é avaliar a taxa de reganho ponderal em um grupo de pacientes submetidos à cirurgia bariátrica.

\section{Material e métodos}

Através da análise de prontuários, recrutamos 35 pacientes (29 mulheres e 6 homens) com idade de 39,3 \pm 9,10 anos que já haviam sido submetidos a algum procedimento bariátrico, independente do tempo decorrido da cirurgia. Os pacientes atendidos no ambulatório de bariátricos da Policlínica Piquet Carneiro (PPC-UERJ) foram convocados através da veiculação de um anúncio em publicação de jornal, o qual convidava aqueles pacientes submetidos à cirurgia bariátrica que estavam sem acompanhamento a reiniciar seu tratamento por equipe especializada. O período de inserção dos pacientes no ambulatório ocorreu entre os meses de abril e setembro de 2013 e a aquisição dos dados do estudo foi feita através de análise do prontuário com os dados das consultas de primeira vez.

Nessa consulta, os pacientes foram submetidos à anamnese e exame físico. As medidas em centímetros da circunferência da cintura, o ponto médio entre a última costela e a crista ilíaca, e do quadril, a área de maior diâmetro ao redor do quadril, foram realizadas em duplicata com o paciente 
em posição ortostática pelo mesmo examinador. A altura dos pacientes foi mensurada em duplicata em estadiômetro com escala de 0,5 centímetros e este valor foi considerado para o cálculo do IMC atual e o IMC pré-cirúrgico relatado pelo paciente. $\mathrm{O}$ IMC é definido como o peso em quilogramas sobre o quadrado da altura em metros. $\mathrm{O}$ peso dos pacientes foi mensurado em balança digital (W300, Welmy, Santa Bárbara D’Oeste, SP, Brasil) em duplicata com pacientes em uso de roupas leves e sem sapatos. Além disso, na anamnese foram coletadas informações sobre: peso pré-cirúrgico, peso mínimo atingido no pós-operatório, ganho ponderal em quilogramas no último ano e tipo de cirurgia realizada. Estes dados foram relatados pelos pacientes e não mensurados ou confirmados em prontuários pela equipe médica. Através do relato das duas primeiras variáveis foram calculados o IMC pré-cirúrgico e o IMC mínimo atingido. Todas as cirurgias relatadas pelos pacientes foram realizadas fora do Hospital Universitário Pedro Ernesto.

O cálculo da perda do excesso de peso (PEP) foi realizado através da seguinte fórmula: PEP = (peso pré-operatório relatado - peso mínimo relatado x 100/(peso pré-operatório relatado peso ideal); onde consideramos o peso ideal como um IMC ideal de $25 \mathrm{~kg} / \mathrm{m}^{2} \mathrm{x}$ altura ${ }^{2}$. Já a taxa de reganho $(T \times R)$ foi calculada através da seguinte fórmula: $T x R=$ (peso atual - peso mínimo relatado) x 100/(peso pré-operatório relatado - peso mínimo relatado). Durante a anamnese também foi questionado sobre uso de medicamentos para HAS, DM2 e dislipidemia. Aqueles pacientes que na primeira consulta relataram o uso de pelo menos um desses medicamentos foram considerados portadores dessas patologias, independentemente de nossa indicação médica para a suspensão dessas drogas na consulta de primeira vez. Por fim, com base na conduta da equipe multidisciplinar durante essa consulta, alguns pacientes tiveram a indicação do uso de algum medicamento para promover perda ponderal, sejam eles sacietógenos, inibidores da lipase intestinal, ou ainda agentes com ação central, serotoninérgicos ou de ação na anidrase carbônica, que promovem perda ponderal por ação indireta no mecanismo da fome e saciedade.

A investigação para a possibilidade de ocorrência do transtorno de compulsão alimentar periódico (TCAP) foi realizada por equipe previamente treinada do Núcleo de Assistência e Pesquisa em Transtornos Alimentares (NAPTA) no dia da consulta de primeira vez, através de questionário validado para investigação de TCAP. ${ }^{6}$ Considerou-se um ponto de corte de 16 para alta probabilidade de ocorrência de TCAP. O tempo gasto com atividades físicas programadas também foi inquerido e categorizamos os pacientes em três grupos: sedentários (nenhuma atividade física/sem), hábitos irregulares (1-149 min/sem) e hábitos regulares (> $150 \mathrm{~min} / \mathrm{sem}$, no mínimo em três sessões semanais).

Para investigação do nosso objetivo primário, o tempo decorrido da cirurgia (em meses) foi a variável que determinou a divisão dos pacientes inicialmente em dois grupos: $\leq 1$ ano $(G \leq 1)$ e > 1 ano $(G>1)$. Posteriormente, estes mesmos pacientes foram reagrupados em outros dois novos grupos: $<2$ anos $(G<2)$ e $\geq 2$ anos $(G \geq 2)$ e ainda em $<5$ anos $(G<5)$ e $\geq 5$ anos $(G \geq 5)$.

A análise estatística foi realizada através do programa GraphPad Prism 5 (San Diego, CA, EUA). Os dados são apresentados como média \pm desvio padrão ou frequência em percentuais. A comparação entre os grupos foi realizada pelo teste $\mathrm{t}$ de Student não pareado. Um nível de significância em $p<0,05$ foi considerado como estatisticamente significante.

\section{Resultados}

Dos 35 pacientes recrutados, 94,3\% ( $\mathrm{n}=$ 33) relataram que foram submetidos à cirurgia de gastroplastia redutora com reconstrução em $Y$ de Roux, enquanto 5,7\% $(n=2)$ dos pacientes referiram a realização das técnicas de derivação biliodigestiva de Scopinaro ou de gastrectomia vertical em manga. É importante ressaltar que todos os pacientes que nos procuraram estavam sem qualquer acompanhamento médico especializado no pós-operatório da cirurgia bariátrica. $\mathrm{O}$ IMC pré-cirúrgico relatado era de 50,5 $\pm 8,92 \mathrm{~kg} /$ $\mathrm{m}^{2}$. Em relação ao tempo decorrido da cirurgia, observamos que $22,8 \%(n=8)$ da amostra havia realizado a cirurgia em um prazo igual ou inferior a um ano, 40\% ( $n=14)$ em menos de dois anos e $74,3 \%(n=26)$ em menos de cinco anos. Na consulta de primeira vez foi possível observar que 68,6\% $(n=24)$ eram sedentários, enquanto 14,3\% ( $n=5)$ 
relataram hábitos irregulares de exercício físicos e somente $17,1 \%(n=6)$ aderiram a um programa de exercícios físicos programados e regulares.

Através do questionário para avaliação de TCAP empregado na rotina do ambulatório foi possível observar que $82,8 \%(n=28)$ apresentavam resultados negativos para esse transtorno. A decisão médica de iniciar algum medicamento com intuito de promover redução de peso e/ou controle de compulsão alimentar foi tomada em $20 \%(n=7)$ da amostra.

Com base na altura aferida na primeira consulta e no peso mínimo relatado pelos pacientes foi possível quantificar o suposto IMC mínimo alcançado no pós-operatório $(30,7 \pm 6,6 \mathrm{~kg} /$ $\mathrm{m}^{2}$ ). Como uma parte substancial de pacientes já referia reganho ponderal $(71,4 \%, \mathrm{n}=25)$, o IMC atual dos pacientes era de $34,2 \pm 6,89 \mathrm{~kg} / \mathrm{m}^{2}$. Em relação às circunferências da cintura e do quadril, estas eram respectivamente de 101,9 \pm 18,2 $\mathrm{cm}$ e 117,2 \pm 14,3 cm. Em relação à ocorrência de comorbidades observadas na primeira consulta, notamos que $28,6 \%(n=10), 5,7 \%(n=2)$ e $3,5 \%(n$ =1) mantinham-se em uso de medicamentos para o controle de HAS, DM2 e dislipidemia, respectivamente. No grupo total foi possível notar que a PEP foi de $79,5 \pm 21,3 \%$ e a TxR de $16,7 \pm 20,4 \%$.

Em relação ao tempo cirúrgico, observamos que a PEP foi maior no grupo $\mathrm{G}>1$ que no grupo $\mathrm{G}$ $\leq 1(85,9 \pm 3,2$ vs. $57,4 \pm 7,5 \% ; p<0,01)$; ocorrendo o mesmo com o grupo $\mathrm{G} \geq 2$ em relação ao $\mathrm{G}<2$ $(88,3 \pm 3,8$ vs. $66,2 \pm 5,3 \% ; p=0,001)$. Entretanto, apesar dos pacientes apresentarem altas taxas de PEPs, ao separá-los em mais ou menos de cinco anos da cirurgia respectivamente, não se observa mais diferença nesta variável $(90,8 \pm 7,8$ vs. 75,5 $\pm 3,8 \%$; NS - figura 1 ).

A TxR já apresenta diferença após o primeiro ano de cirurgia. $O$ grupo $G \leq 1$ apresentou uma TxR menor que o $G>1$ (3,8 \pm 3,1 vs. 20,6 \pm 4,1\%; $p$ $<0,05)$. Tal fato se acentua após o segundo ano da cirurgia $(2,6 \pm 1,8$ vs. $26,2 \pm 4,6 \% ; P<0,001)$; tornando-se ainda mais relevante após cinco anos da cirurgia (10,1 $\pm 3,0$ vs. 35,9 $\pm 7,2 \%$; $p<$ 0,001 - figura 2).

Em valores absolutos, foi possível quantificar o suposto ganho ponderal relatado em quilos no último ano pelos pacientes. Em relação aos que se submeteram à cirurgia há mais de cinco anos houve uma importante diferença se compararmos àqueles com menos de cinco anos da cirurgia no ganho absoluto de peso relatado no último ano $(7,5 \pm 1,7$ vs. $2,4 \pm 0,9 \mathrm{~kg} ; \mathrm{p}<0,01)$.

\section{Discussão}

A cirurgia bariátrica é uma importante opção terapêutica para pacientes com obesidade, existindo diferentes técnicas cirúrgicas na atualidade. A mais frequentemente empregada é a gastroplastia redutora com reconstrução em $Y$ de Roux, ${ }^{7}$ que correspondeu à técnica realizada em $94,3 \%$ dos pacientes que participaram de nosso estudo. Essa modalidade é considerada por alguns autores como padrão-ouro, sendo a mais utilizada em todo mundo. ${ }^{8} \mathrm{O}$ tratamento cirúrgico da obesidade é considerado efetivo, no entanto, observa-se dificuldade de manutenção da perda ponderal, além de altas taxas de reganho ponderal pós-cirúrgico, o que pode trazer consequências psicossociais, médicas e ainda econômicas não só para o paciente, mas também para a sociedade., ${ }^{5,9}$

Diferentes fatores podem influenciar na evolução da perda de peso e na taxa de reganho como, por exemplo, fatores sociais, psicológicos, presença de comorbidades associadas, técnica cirúrgica empregada, IMC pré-cirúrgico e aderência ao acompanhamento pós-cirúrgico. ${ }^{10}$ Dos 35 pacientes avaliados, o IMC pré-cirúrgico médio relatado foi de $50,5 \pm 8,92 \mathrm{~kg} / \mathrm{m}^{2}$, sendo estes dados semelhantes aos encontrados no estudo da Sociedade Americana de Cirurgia Bariátrica, ${ }^{1}$ no qual a média de IMC pré-cirúrgico entre as mulheres foi de $46,5 \pm 7,1 \mathrm{~kg} / \mathrm{m}^{2}$ e nos homens $50,4 \pm 7,1 \mathrm{~kg} / \mathrm{m}^{2}$. Estudos prévios relatam que há uma associação entre maior IMC inicial e menor perda de peso ao longo do acompanhamento. Além disso, está comprovado que a mudança de estilo de vida está relacionada com melhor evolução pós-cirúrgica. Dados da literatura mostram que após a realização do procedimento cirúrgico, há um aumento na prática de atividade física. ${ }^{12}$ No entanto, em nosso estudo, a maioria dos pacientes $(82,9 \%)$ relatou ser sedentário ou praticar irregularmente exercícios físicos. Não está totalmente esclarecido porque alguns pacientes mantêm-se sedentários e outros não, no entanto, uma das teorias é sobre falta de conhecimento específico sobre os benefícios do exercício físico para a saúde humana. Wouters e colaboradores ${ }^{12}$ suge- 
rem que a segmentação do conhecimento sobre o exercício antes e após a cirurgia pode levar a uma melhora relevante na aderência à atividade física. Muitas vezes os pacientes acreditam que a cirurgia bariátrica representa a "cura" da obesidade e se posicionam de maneira passiva, não considerando necessário e importante adotar outras medidas comportamentais..$^{13}$ Além disso, devemos ressaltar que nenhum paciente que nos procurou estava em acompanhamento médico especializado, independentemente do tempo decorrido da cirurgia. Tal fato, além de ressaltar que muitos acreditam que a cirurgia é ponto crucial e fundamental para a cura de sua doença, enfatiza também que a não aderência desses pacientes ao acompanhamento multiprofissional não permite que mudanças em seu estilo de vida sejam estimuladas e orientadas.

Dados da literatura são conflitantes quando correlacionam TCAP e cirurgia bariátrica. Alguns estudos apontam para um aumento de TCAP em pacientes bariátricos, contudo, em nossa amostra a maioria dos pacientes apresentou resultados negativos. Outros trabalhos, como o de Burgmer e colaboradores $^{14}$, não apoiam o impacto da presença do TCAP na evolução pós-cirúrgica de obesos, sendo assim, mais pesquisas são necessárias para esclarecer essa relação. ${ }^{15}$ Devemos também ressaltar que não há questionário validado para essa investigação em bariátricos e o uso deste método pode ser questionado nessa população.
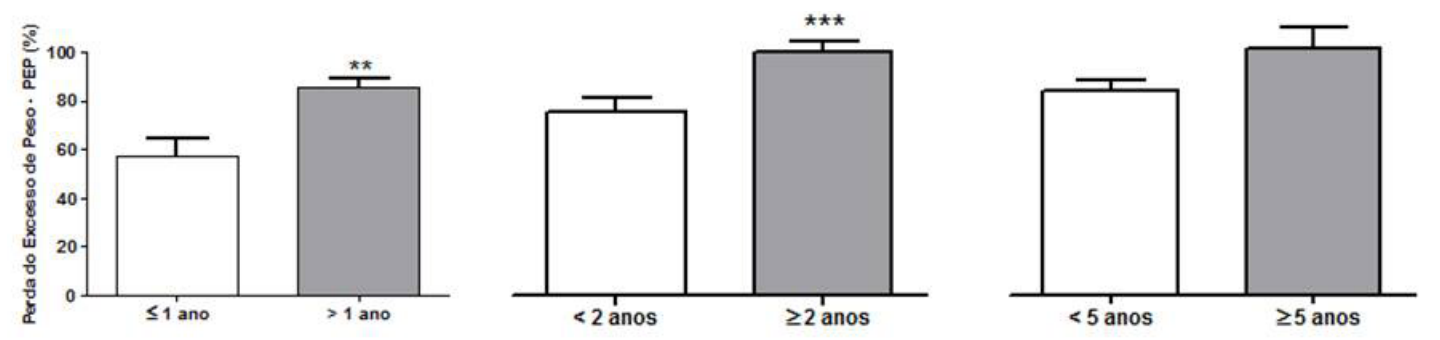

Figura 1: Perda do excesso de peso (PEP) de acordo com o tempo decorrido da cirurgia. ${ }^{* * P}<0,01 ; * * * P<0,001$.
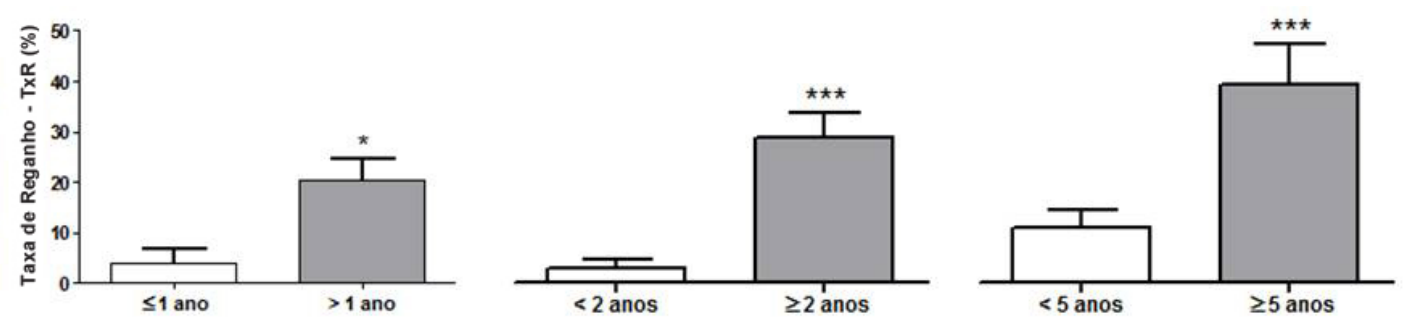

Figura 2: Taxa de reganho ponderal (TxR) de acordo com o tempo decorrido da cirurgia. ${ }^{*} \mathrm{P}<0,05 ;{ }^{* * *} \mathrm{P}<0,001$. 
o tempo decorrido da cirurgia.

A etiologia do reganho de peso é complexa e multifatorial. Dados da literatura mostram que transgressão alimentar (25,3\%), sedentarismo (21\%) e falta de motivação (19,7\%) estão entre as principais causas. ${ }^{18}$ Os resultados encontrados em nosso estudo podem ser justificados pela característica da amostra, já que foram analisados pacientes que estavam sem acompanhamento especializado pós-cirúrgico. Isso porque a literatura mostra que pacientes sem acompanhamento apresentam 4,6 vezes maior reganho de peso do que pacientes que seguem o acompanhamento clínico corretamente. ${ }^{9}$ Outros estudos mostram que perda de acompanhamento após a cirurgia está relacionada com aumento da taxa de reganho e de complicações pós-operatórias. ${ }^{5,9,10}$ Fatores como IMC prévio alto e sedentarismo também estão relacionados com taxa de reganho crescente e perda de excesso de peso cada vez menor., ${ }^{5,12}$

As limitações deste estudo estão relacionadas com o relato não comprovado de algumas variáveis analisadas, já que estes pacientes foram acompanhados no período pré-cirúrgico e submetidos à cirurgia em outras unidades médicas.

Infelizmente, a existência de pacientes bariátricos sem qualquer acompanhamento por equipe multiprofissional já é uma realidade em nosso meio, e a recidiva do excesso de peso tem importante consequência clínica, psicossocial e econômica. Dessa forma, é importante que se entenda as diferentes causas relacionadas com o reganho ponderal pós-cirúrgico, para que se possa desenvolver estratégias de manejo e prevenção pré e pós-operatórias com equipes multidisciplinares treinadas para o manejo clínico destes pacientes.

\section{Referências}

1. Zeve JLM, Novais PO, Oliveira Jr N. Técnicas em cirurgia bariátrica: uma revisão da literatura. Rev Ciênc Saúde. 2012;5(2):132-40.

2. Instituto Brasileiro de Geografia e Estatística. Antropometria e estado nutricional de crianças, adolescentes e adultos no Brasil. Rio de Janeiro: IBGE; 2008-2009. Disponível em: http://www. ibge.gov.br/home/presidencia/noticias/imprensa/ ppts/0000000108.pdf.

3. Kubik JF, Gill RS, Laffin M, Karmali S. The Impact of Bariatric Surgery on Psychological
Health. J Obes. 2013;2013:837989. http://dx.doi. org/10.1155/2013/837989

4. Segal A, Fandiño J. Indicações e contraindicações para realização das operações bariátricas. Rev Bras Psiquiatr. 2002;24(Supl III):68-72.

5. Karmali S, Brar B, Shi X, Sharma AM, Gara C, Birch DW. Weight Recidivism Post-Bariatric Surgery: A Systematic Review. Obes Surg. 2013 Nov;23(11):192233. http://dx.doi.org/10.1007/s11695-013-1070-4

6. Freitas S, Gorenstein C, Apolinario JC. Instrumentos para avaliação dos transtornos alimentares. Rev Bras Psiquiatr. 2011 Dec;23(4):215-20. http://dx.doi. org/10.1590/S1516-44462002000700008

7. Farias PM, Furtado CAS, Morales G, Santos LC, Coutinho V. Compulsão alimentar em pacientes submetidos à cirurgia bariátrica. Rev Bras Nutr Clin. 2009 Feb;24(3):137-42.

8. Fobi LA, Lee H, Felahy B, Che K, Ako P, Fobi N. Choosing an operation for weight control, and the transected banded gastric bypass. Obes Surg. 2005 Jan;15(1):114-21.

9. Sjöström L, Lindroos AK, Peltonen M, Torgerson J, Bouchard C, Carlsson B, et al. Lifestyle, diabetes, and cardiovascular risk factors 10 years after bariatric surgery. N Engl J Med. 2004 Dec;351(26):2683-93. http://dx.doi.org/10.1056/NEJMoa035622

10. Villa AG, López AMS, Calleja KGM, Gómez LML, Gómez JD, Escobar JMT. Factores relacionados con la pérdida de peso en una cohorte de pacientes obesos sometidos a bypass gástrico. Nutr Hosp. 2013;28(3):623-30. http://dx.doi.org/10.3305/ nh.2013.28.3.6176

11. Renquist K. Obesity classification. Obes Surg. 1998;8(4):480.

12. Wouters EJ, Larsen JK, Zijlstra H, van Ramshorst B, Geenen R. Physical activity after surgery for severe obesity: the role of exercise cognitions. Obes Surg. 2011 Dec;21(12):1894-9. http://dx.doi.org/10.1007/ s11695-010-0276-y

13. Sjöstrom L, Narbro K, Sjöstrom CD, Karason K, Larsson B, Wedel H, et al. Effects of Bariatric Surgery on Mortality in Swedish Obese Subjects. N Engl J Med. 2007 Aug;357(8):741-52. http://dx.doi. org/10.1056/NEJMoa066254

14. Burgmer R, Grigutsch K, Zipfel S, Wolf AM, de Zwaan M, Husemann B, et al. The influence of eating behavior and eating pathology on weight loss after gastric restriction operations. Obes Surg. 2005;15(5):684-91.

15. Busetto L, Segato G, De Luca M, De Marchi F, Foletto $\mathrm{M}$, Vianello $\mathrm{M}$, et al. Weight loss and postoperative complications in morbidly obese patients with binge eating disorder treated by laparoscopic adjustable gastric banding. Obes Surg. 2005 Feb;15(2):195-201. 
16. Sjöström CD, Lissner L, Wedel H, Sjöström L. Reduction in incidence of diabetes, hypertension and lipid disturbances after intentional weight loss induced by bariatric surgery: the SOS Intervention Study. Obes Res. 1999 Sep;7(5):477-84.

17. Sugerman HJ, Sugerman EL, DeMaria EJ, Kellum JM, Kennedy C, Mowery Y, et al. Bariatric surgery for severely obese adolescents. J Gastrointest Surg. 2003;7(1):102-8.

18. Odom J, Zalesin KC, Washington TL, Miller WW, Hakmeh B, Zaremba DL, et al. Behavioral predictors of weight regain after bariatric surgery. Obes Surg. 2010 Mar;20(3):349-56. http://dx.doi.org/10.1007/ s11695-009-9895-6

Recebido: $\quad$ 21/11/2013.

Revisado: $\quad$ 26/11/2013.

Aprovado: $\quad$ 04/02/2014. 